\title{
Kinetische und mechanistische Untersuchungen von Übergangsmetall-Komplex-Reaktionen
}

\section{XXIII *. Kinetik und Mechanismus der Reaktion von 1-Diethylamino-1-propin mit übergangsmetallkoordinierten Selenoketonen}

Helmut Fischer ${ }^{\star}$, Ulrike Gerbing und Athanassios Tiriliomis

Anorganisch-chemisches Institut der Technischen Universität München, Lichtenbergstr. 4, D-8046 Garching (B.R.D.)

\begin{abstract}
The pentacarbonyl(arylphenylselenoketone) complexes $(\mathrm{CO})_{5} \mathrm{M}[\mathrm{Se}=\mathrm{C}(\mathrm{Ph})-$ $\left.\mathrm{C}_{6} \mathrm{H}_{4} \mathrm{R}-p\right]$ ] $\left(\mathrm{M}=\mathrm{Cr}, \mathrm{R}=\mathrm{H}\right.$ (Ia); $\mathrm{M}=\mathrm{W}, \mathrm{R}=\mathrm{H}$ (Ib), $\mathrm{Br}$ (Ic), OMe (Id), $\mathrm{NMe}_{2}$ (Ie)) react with 1-diethylamino-1-propyne, $\mathrm{Et}_{2} \mathrm{NC} \equiv \mathrm{CMe}$ (II), via [2 + 2]-cycloaddition across the $\mathrm{C} \equiv \mathrm{C}$ triple bond of II to the $\mathrm{Se}=\mathrm{C}$ bond of $\mathrm{I}$ and electrocyclic ring-opening to give selenoacrylamide complexes, $(\mathrm{CO})_{5} \mathrm{M}\left[\mathrm{Se}=\mathrm{C}\left(\mathrm{NEt}_{2}\right) \mathrm{C}(\mathrm{Me})=\right.$ $\left.\mathrm{C}(\mathrm{Ph}) \mathrm{C}_{6} \mathrm{H}_{4} \mathrm{R}-\mathrm{p}\right]$ (III). The reaction follows second-order rate kinetics: $-\mathrm{d}[\mathrm{I}] / \mathrm{d} t=$ $k_{2}[I][I I]$. For the reaction of I with II in toluene, the activation enthalpy $\Delta H^{*}$ is $29(1) \mathrm{kJ} / \mathrm{mol}$ for both $\mathrm{Ia}$ and $\mathrm{Ib}$, the activation entropy $\Delta S^{*}$ is $-152(5) \mathrm{J} /(\mathrm{mol} \mathrm{K})$ for Ia and $-161(2) \mathrm{J} /(\mathrm{mol} \mathrm{K})$ for Ib. The rate constant $k_{2}$ decreases clearly with increasing donor capacity of $\mathbf{R}$ in $\mathrm{Ib}-\mathrm{e}$. A good positive correlation is observed between $k_{2}$ and the Hammett constants $\sigma_{\mathrm{p}}$ of the $\mathrm{R}$ with $\rho=2.50$. In contrast, the influence of the metal and the solvent is small. At $20.6^{\circ} \mathrm{C}, k_{2}(\mathrm{~W})$ is only 3.48 $k_{2}(\mathrm{Cr})$ (for $\mathrm{R}=\mathrm{H}$ in toluene) and $k_{2}$ (nitrobenzene) $=6.8 k_{2}$ (decane). A concerted biradicaloid mechanism with an early transition state is proposed. Reaction of the $\mathrm{Se}=\mathrm{C}$ bond of I with 1-diethylamino-1-propyne is compared with the reaction of $\mathrm{W}=\mathrm{C}, \mathrm{O}=\mathrm{C}$ and $\mathrm{S}=\mathrm{C}$ bonds with the same alkyne.
\end{abstract}

\section{Zusammenfassung}

Die Pentacarbonyl(arylphenylselenoketon)-Komplexe $(\mathrm{CO})_{5} \mathrm{M}\left[\mathrm{Se}=\mathrm{C}(\mathrm{Ph}) \mathrm{C}_{6} \mathrm{H}_{4} \mathrm{R}\right.$ p] $\left(\mathrm{M}=\mathrm{Cr}, \mathrm{R}=\mathrm{H}\right.$ (Ia); $\mathrm{M}=\mathrm{W}, \mathrm{R}=\mathrm{H}$ (Ib), $\mathrm{Br}$ (Ic), OMe (Id), $\mathrm{NMe}_{2}$ (Ie))

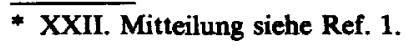


reagieren mit 1-Diethylamino-1-propin, $\mathrm{Et}_{2} \mathrm{NC} \equiv \mathrm{CMe}$ (II), unter $[2+2]-C y c l o-$ addition der $\mathrm{C} \equiv \mathrm{C}$-Dreifachbindung von II an die $\mathrm{Se}=\mathrm{C}$-Bindung von I und elektrocyclischer Ringöffnung zu Selenoacrylamid-Komplexen, $(\mathrm{CO})_{5} \mathrm{M}\left[\mathrm{Se}=\mathrm{C}\left(\mathrm{NEt}_{2}\right)\right.$ $\mathrm{C}(\mathrm{Me})=\mathrm{C}(\mathrm{Ph}) \mathrm{C}_{6} \mathrm{H}_{4} \mathrm{R}-\mathrm{p}$ ] (III). Die Reaktion erfolgt nach einem Geschwindigkeitsgesetz zweiter Ordnung: $-\mathrm{d}[\mathrm{I}] / \mathrm{d} t=k_{2}[\mathrm{I}][\mathrm{II}]$. Für die Reaktion von I mit II in Toluol beträgt die Aktivierungsenthalpie $\Delta H^{\neq} 29(1) \mathrm{kJ} / \mathrm{mol}$ (Ia und Ib), die Aktivierungsentropie $\Delta S^{\neq}-152(5) \mathrm{J} /(\mathrm{mol} \mathrm{K})$ (la) bzw. $-161(2) \mathrm{J} /(\mathrm{mol} \mathrm{K})$ (Ib). Mit zunehmender Donorfähigkeit von $\mathrm{R}$ in $\mathrm{Ib}-\mathrm{e}$ nimmt die Geschwindigkeitskonstante $k_{2}$ deutlich ab. Zwischen $k_{2}$ und den Hammett-Konstanten $\sigma_{\mathrm{p}}$ für $\mathbf{R}$ besteht eine gute positive Korrelation $(\rho=2.50)$. Im Gegensatz dazu ist der Einfluss des Metalls und des Lösungsmittels gering. Bei $20.6^{\circ} \mathrm{C}$ ist $k_{2}(\mathrm{~W})$ nur $3.48 k_{2}(\mathrm{Cr})$ (für $\mathrm{R}=\mathrm{H}$ in Toluol) und $k_{2}$ (Nitrobenzol) $=6.8 k_{2}$ (Decan). Ein konzertierter biradikaloider Mechanismus mit einem frühen Übergangszustand wird vorgeschlagen. Die Reaktion der Se=C-Bindung von I mit 1-Diethylamino-1-propin wird mit derjenigen von $\mathrm{W}=\mathrm{C}-, \mathrm{O}=\mathrm{C}-\mathrm{bzw} . \mathrm{S}=\mathrm{C}-\mathrm{Bindungen}$ mit dem gleichen Alkin verglichen.

Selenobenzaldehyde und Diarylselenoketone sind monomer in freier Form nicht stabil [2], lassen sich aber als hochreaktive Spezies erzeugen und in situ durch Reaktion mit konjugierten Dienen als Diels-Alder-Cycloaddukte abfangen [3]. Im Gegensatz dazu sind Übergangsmetallkomplexe von Selenoaldehyden und Selenoketonen weitgehend beständig [4,5]. Im Fall der Pentacarbonylchrom- und -wolfram-Komplexe wird die extrem hohe Reaktivität der freien SelenocarbonylVerbindungen durch die Koordination an das Metall jedoch nicht vollständig aufgehoben, sondern nur reduziert. Dies zeigt sich z.B. in deren bereitwilliger Reaktion mit 1,3-Dienen, die zu metallkoordinierten Selenacyclen führt [6].

Mit Inaminen wie 1-Diethylamino-1-propin reagieren an Pentacarbonylchromoder -wolfram gebundene Selenoaldehyde und -ketone rasch regio- und - im Fall der Selenoaldehyd-Komplexe - auch stereospezifisch $\mathrm{zu}$ metallkoordinierten Selenoacrylamidderivaten (G1. 1) [7].

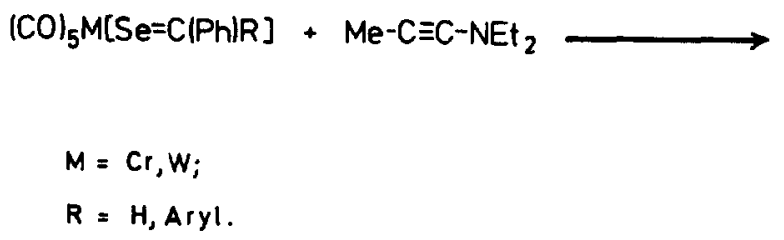

Unter CO-Druck lassen sich die Selenoacrylamide schonend und nahezu quantitativ von Metall ablösen. Bei Verwendung von Selenoaldehyd-Komplexen $(R=H)$ erhält man dabei das $E$-konfigurierte Selenoacrylamid [7].

Die Bildung der Selenoacrylamid-Komplexe lässt sich im Prinzip unter der Annahme einer regiospezifischen Addition der $\mathrm{C} \equiv \mathrm{C}$-Dreifachbindung das Aminoalkins an die Se=C-Doppelbindung des Komplexes unter Bildung eines metallkoordinierten Selenetens und nachfolgender elektrocyclischer Ringöffnung verstehen. Eine detaillierte kinetische Untersuchung der Reaktion von 1-Diethylamino-1-propin (II) mit Pentacarbonyl(diphenylselenoketon)chrom (Ia) sowie mit einer Reihe von para-substituierten Pentacarbonyl(arylphenylselenoketon)wolfram- 
Komplexen (Ib-e) sollte nun nähere Aufschlüsse über den Reaktionsablauf liefern.

\section{Kinetische Untersuchungen}

Die Umsetzungen der Komplexe I mit dem Inamin II (Gl. 2) verlaufen bereits bei geringem Inamin-Überschuss quantitativ.

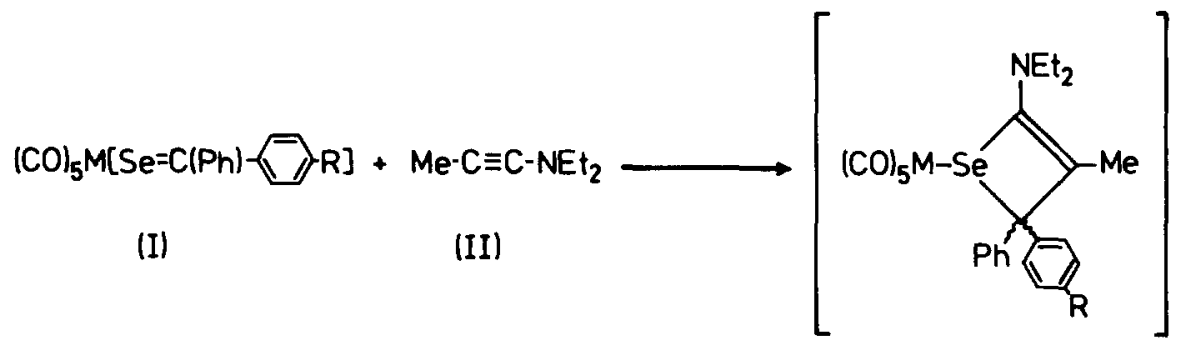

(A)

\begin{tabular}{c||cc} 
I,III & M & R \\
\hline \hline a & Cr & H \\
b & W & H \\
c & W & Br \\
d & W & OMe \\
e & W & NMe2
\end{tabular}

(2)<smiles>[R]c1ccc(C(=C(C)C(N=C)=[Se]C(C)(C)C)c2ccccc2)cc1</smiles>

(III)

Die Selenoketon-Komplexe I sind intensiv blau, die resultierenden Selenoacrylamid-Komplexe III hingegen gelb gefärbt. Die Reaktion von I mit II lässt sich somit gut anhand der Intensitätsabnahme der charakteristischen MLCT-Bande von I (Absorptionsmaximum im Bereich zwischen 595 und $630 \mathrm{~nm}$, geringfügig abhängig von $M$ und $R$ ) verfolgen.

Die Messungen wurden unter Bedingungen pseudo-erster Ordnung durchgeführt. Beim Chrom-Komplex Ia wurde das Verhältnis [II $]_{0} /$ [Komplex $]_{0}$ zwischen 50 und 730 , bei den Wolfram-Komplexen Ib-e zwischen 11 und 2370 - abhängig von $R$ und der Art des Solvens - variiert.

Die Geschwindigkeitskonstanten $k_{\mathrm{ps}}$ in Toluol sind unabhängig von der Anfangskonzentration des Komplexes, $[\mathrm{I}]_{0}$, und nehmen mit steigender Inaminkonzentration linear zu (Tabellen 1 und 2, Fig. 1). Die Reaktion lässt sich somit durch ein Geschwindigkeitsgesetz zweiter Ordnung, jeweils von erster Ordnung bezüglich des Komplexes und des Inamins, beschreiben: $-\mathrm{d}[\mathrm{I}] / \mathrm{d} t=k_{2}[\mathrm{I}][\mathrm{II}]$; mit $k_{\mathrm{ps}}=k_{2}$ [II]. Die Geschwindigkeitskonstanten zweiter Ordnung $k_{2}$ sind in Tabelle 3 zusammengestellt. 
Tabelle 1

Geschwindigkeitskonstanten pseudo-erster Ordnung $k_{\mathrm{ps}}$ für die Reaktion des Chrom-Komplexes (CO) ${ }_{5} \mathrm{Cr}\left[\mathrm{Se}=\mathrm{CPh}_{2}\right.$ ] (Ia) mit 1-Diethylamino-1-propin (II) in Toluol

\begin{tabular}{|c|c|c|c|}
\hline $\begin{array}{l}\text { Temp. } \\
\left({ }^{\circ} \mathrm{C}\right)\end{array}$ & $\begin{array}{l}{[\mathrm{Komp}]_{0}} \\
(\mathrm{mmol} / \mathrm{l})\end{array}$ & $\begin{array}{l}{[\mathrm{II}]} \\
(\mathrm{mmol} / \mathrm{I})\end{array}$ & $\begin{array}{l}10^{3} \cdot k_{\mathrm{ps}} \\
\left(\mathrm{s}^{-1}\right)\end{array}$ \\
\hline \multirow[t]{4}{*}{16.9} & 0.08 & 8.44 & 4.29 \\
\hline & 0.08 & 16.9 & 8.38 \\
\hline & 0.08 & 25.3 & 12.5 \\
\hline & 0.08 & 33.8 & 17.6 \\
\hline \multirow[t]{6}{*}{20.6} & 0.05 & 12.2 & 7.75 \\
\hline & 0.10 & 12.2 & 7.76 \\
\hline & 0.20 & 12.2 & 7.96 \\
\hline & 0.10 & 18.2 & 12.1 \\
\hline & 0.10 & 36.5 & 24.3 \\
\hline & 0.10 & 73.0 & 46.2 \\
\hline \multirow[t]{4}{*}{24.8} & 0.08 & 7.55 & 5.83 \\
\hline & 0.08 & 15.1 & 11.3 \\
\hline & 0.08 & 22.7 & 17.1 \\
\hline & 0.08 & 30.2 & 23.6 \\
\hline \multirow[t]{3}{*}{29.0} & 0.10 & 5.22 & 4.62 \\
\hline & 0.10 & 12.6 & 12.0 \\
\hline & 0.10 & 20.9 & 18.8 \\
\hline \multirow[t]{4}{*}{33.9} & 0.08 & 8.71 & 9.20 \\
\hline & 0.08 & 17.4 & 18.2 \\
\hline & 0.08 & 26.1 & 28.0 \\
\hline & 0.08 & 34.9 & 36.1 \\
\hline \multirow[t]{3}{*}{37.7} & 0.10 & 5.03 & 6.73 \\
\hline & 0.10 & 12.6 & 15.9 \\
\hline & 0.10 & 20.1 & 26.0 \\
\hline
\end{tabular}

Aus der Temperaturabhängigkeit der Geschwindigkeitskonstanten errechnen sich die Aktivierungsparameter in Toluol nach der Eyring-Gleichung für die Reaktion des Chrom-Komplexes Ia mit II zu (Standardabweichung in Klammern): $\Delta \boldsymbol{H}^{\neq}=$ 29(1) $\mathrm{kJ} / \mathrm{mol} ; \Delta S^{*}=-152(5) \mathrm{J} /(\mathrm{mol} \mathrm{K})$ und für die Reaktion des WolframKomplexes Ib mit II zu $\Delta H^{*}=29(1) \mathrm{kJ} / \mathrm{mol} ; \Delta S^{\star}=-161(2) \mathrm{J} /(\mathrm{mol} \mathrm{K})$.

Der jeweils sehr niedrige Wert für die Aktivierungsenthalpie und die stark negativen Aktivierungsentropien lassen auf einen geschwindigkeitsbestimmenden assoziativen Reaktionsschritt schliessen.

Die Geschwindigkeitskonstante $k_{2}$ ist stark abhängig vom para-ständigen Substituenten R. Mit zunehmender Fähigkeit von $R$, die Elektronendichte im Ring und damit auch in der $\mathrm{C}=$ Se-Bindung zu erhöhen, nimmt die Geschwindigkeitskonstante deutlich ab: bei $20.6^{\circ} \mathrm{C}$ ist $k_{2}$ für den $p$-Brom-Komplex Ic $363 \mathrm{mal}$ grösser als für die $p$-Dimethylaminoverbindung Ie. Zwischen der Geschwindigkeitskonstanten $k_{2}$ und den Hammett-Konstanten $\sigma_{p}$ für die unterschiedlichen para-ständigen Substituenten $\mathbf{R}$ besteht ein linearer positiver Zusammenhang (Korrelationskoeffizient: 0.993) (Fig. 2) mit $\rho=2.50$.

Vergleichsweise gering ist hingegen der Einfluss des Zentralmetalls und des Lösungsmittels. So reagiert der Wolfram-Komplex Ib bei $20.6^{\circ} \mathrm{C}$ nur $3.48 \mathrm{mal}$ 
Tabelle 2. Geschwindigkeitskonstanten pseudo-erster Ordnung $k_{\mathrm{ps}}$ fir die Reaktion der WolframKomplexe (CO) $)_{5} \mathrm{~W}\left[\mathrm{Se}=\mathrm{C}\left(\mathrm{C}_{6} \mathrm{H}_{4} \mathrm{R}-\mathrm{p}\right) \mathrm{Ph}\right](\mathrm{Ib}-\mathrm{e})$ mit 1-Diethylamino-1-propin (II) $\left.(\mathrm{II}]_{0} 8 \times 10^{-5} \mathrm{~mol} / \mathrm{l}\right)$

\begin{tabular}{|c|c|c|c|c|}
\hline $\mathbf{R}$ & Solvens ${ }^{2}$ & $\begin{array}{l}\text { Temp. } \\
\left({ }^{\circ} \mathrm{C}\right)\end{array}$ & $\begin{array}{l}{[I I]} \\
(\mathrm{mmol} / \mathrm{l})\end{array}$ & $\begin{array}{l}10^{3} \cdot k_{\mathrm{ps}} \\
\left(\mathrm{s}^{-1}\right)\end{array}$ \\
\hline$\overline{\mathbf{H}}$ & Tol & 16.9 & $\begin{array}{r}2.92 \\
4.38 \\
5.84 \\
8.76 \\
11.7\end{array}$ & $\begin{array}{c}5.50 \\
8.00 \\
11.0 \\
16.1 \\
22.7\end{array}$ \\
\hline II & Tol & 25.0 & $\begin{array}{r}3.18 \\
6.36 \\
9.55 \\
12.7\end{array}$ & $\begin{array}{l}7.45 \\
15.2 \\
22.7 \\
31.5\end{array}$ \\
\hline $\mathbf{H}$ & Tol & 29.0 & $\begin{array}{l}5.22 \\
10.4 \\
15.7 \\
20.9\end{array}$ & $\begin{array}{l}14.6 \\
30.0 \\
43.5 \\
60.0\end{array}$ \\
\hline $\mathbf{H}$ & Tol & 37.7 & $\begin{array}{c}2.53 \\
5.05 \\
7.55 \\
10.1\end{array}$ & $\begin{array}{c}8.85 \\
17.6 \\
28.5 \\
37.7\end{array}$ \\
\hline $\mathbf{H}$ & Tol & 46.2 & $\begin{array}{l}1.80 \\
3.61 \\
5.41 \\
7.21\end{array}$ & $\begin{array}{c}8.24 \\
17.9 \\
25.6 \\
35.7\end{array}$ \\
\hline $\mathrm{Br}$ & Tol & 20.6 & $\begin{array}{c}2.93 \\
5.87 \\
17.6 \\
23.5\end{array}$ & $\begin{array}{l}11.4 \\
23.0 \\
69.3 \\
92.9\end{array}$ \\
\hline OMe & Tol & 20.6 & $\begin{array}{l}2.93 \\
23.5 \\
34.8\end{array}$ & $\begin{array}{c}0.944 \\
7.56 \\
12.1\end{array}$ \\
\hline $\mathrm{NMe}_{2}$ & Tol & 20.6 & $\begin{array}{r}23.5 \\
69.3 \\
94.8 \\
190.0\end{array}$ & $\begin{array}{l}0.221 \\
0.745 \\
1.00 \\
2.06\end{array}$ \\
\hline $\mathbf{H}$ & D & 20.6 & $\begin{array}{l}5.90 \\
11.8 \\
23.5 \\
35.3\end{array}$ & $\begin{array}{c}9.34 \\
19.4 \\
38.4 \\
58.0\end{array}$ \\
\hline $\mathbf{H}$ & Tri & 20.6 & $\begin{array}{l}4.41 \\
6.62 \\
8.82\end{array}$ & $\begin{array}{l}19.0 \\
28.7 \\
40.8\end{array}$ \\
\hline $\mathbf{H}$ & Dbu & 20.6 & $\begin{array}{c}3.82 \\
7.65 \\
15.3 \\
23.0\end{array}$ & $\begin{array}{l}10.9 \\
23.0 \\
45.8 \\
64.7\end{array}$ \\
\hline $\mathbf{H}$ & $\mathbf{N}$ & 20.6 & $\begin{array}{l}0.882 \\
2.20 \\
3.53 \\
5.51\end{array}$ & $\begin{array}{c}6.61 \\
19.7 \\
36.0 \\
58.6\end{array}$ \\
\hline
\end{tabular}

"Solvenzien: Toluol (Tol), Decan (D), 1,1,2-Trichlorethan (Tri), Dibutylether (Dbu), Nitrobenzol (N). 


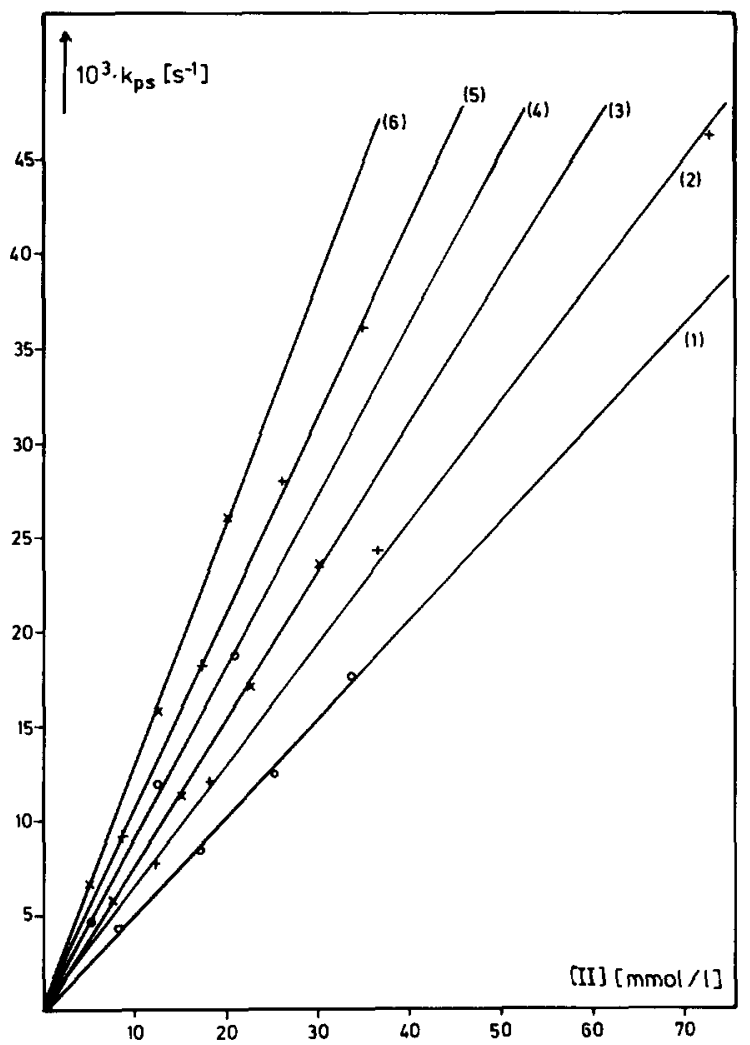

Fig. 1. Abhängigkeit der Geschwindigkeitskonstanten pseudo-erster Ordnung der Reaktion von Ia mit II

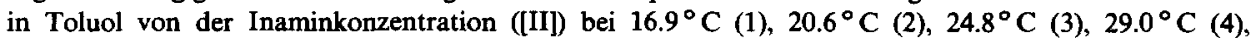
$33.9^{\circ} \mathrm{C} \mathrm{(5)} \mathrm{und} 37.7^{\circ} \mathrm{C}(6)$.

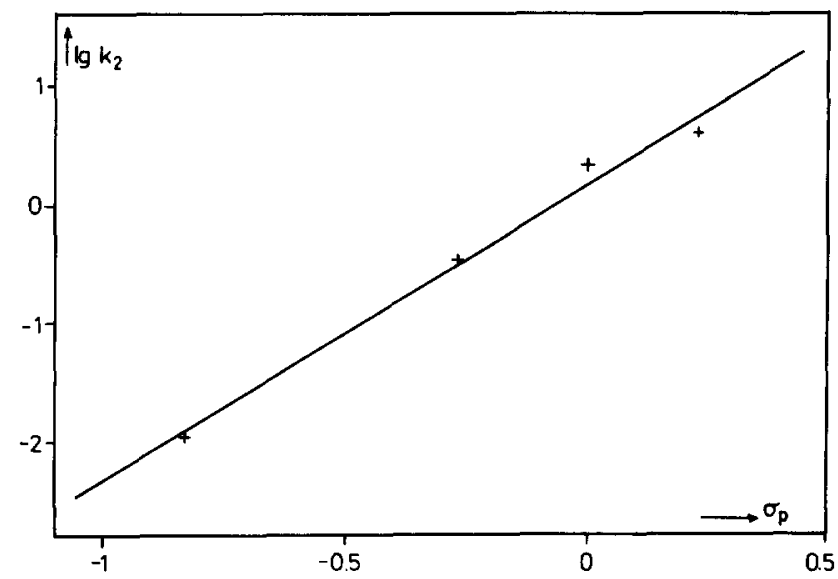

Fig. 2. Zusammenhang zwischen den Geschwindigkeitskonstanten zweiter Ordnung für die Reaktion von $\mathrm{Ib}-\mathrm{e}$ mit II in Toluol bei $20.6^{\circ} \mathrm{C}$ und den Hammett-Konstanten $\sigma_{\mathrm{p}}$. 
Tabelle 3

Geschwindigkeitskonstanten zweiter Ordnung $k_{2}$ für die Reaktion der Diphenylselenoketon-Komplexe I mit 1-Diethylamino-1-propin (II) in Toluol

\begin{tabular}{|c|c|c|c|c|}
\hline$\overline{\mathbf{M}}$ & $\mathbf{R}$ & Solvens $^{a}$ & $\begin{array}{l}\text { Temp. } \\
\left({ }^{\circ} \mathrm{C}\right)\end{array}$ & $\begin{array}{l}k_{2} \\
(\mathrm{l} /(\mathrm{mol} \mathrm{s}))\end{array}$ \\
\hline $\mathrm{Cr}$ & $\mathbf{H}$ & Tol & $\begin{array}{l}16.9 \\
20.6 \\
24.8 \\
29.0 \\
33.9 \\
37.7\end{array}$ & $\begin{array}{l}0.521 \\
0.629 \\
0.782 \\
0.903 \\
1.04 \\
1.28\end{array}$ \\
\hline W & $\mathbf{H}$ & Tol & $\begin{array}{l}16.9 \\
20.6 \\
25.0 \\
29.0 \\
37.7 \\
46.2\end{array}$ & $\begin{array}{l}1.95 \\
2.19^{b} \\
2.51 \\
2.86 \\
3.87 \\
5.00\end{array}$ \\
\hline w & $\begin{array}{l}\mathrm{Br} \\
\mathrm{OMe} \\
\mathrm{NMe}_{2}\end{array}$ & $\begin{array}{l}\text { Tol } \\
\text { Tol } \\
\text { Tol }\end{array}$ & $\begin{array}{l}20.6 \\
20.6 \\
20.6\end{array}$ & $\begin{array}{l}3.96 \\
0.346 \\
0.0109\end{array}$ \\
\hline w & $\mathbf{H}$ & $\begin{array}{l}\text { D } \\
\text { Tri } \\
\text { Dbu } \\
\mathbf{N}\end{array}$ & $\begin{array}{r}20.6 \\
20.6 \\
20.6 \\
20.6\end{array}$ & $\begin{array}{c}1.65 \\
4.94 \\
2.82 \\
11.3\end{array}$ \\
\hline
\end{tabular}

a Solvenzien: Toluol (Tol), Decan (D), 1,1,2-Trichlorethan (Tri), Dibutylether (Dbu), Nitrobenzol (N).

${ }^{b}$ Aus der Temperaturabhängigkeit von $k_{2}$ errechnet.

rascher mit II als der Chrom-Komplex Ia. Mit zunehmender Polarität des Solvens nimmt bei der Reaktion von Ib mit II bei $20.6^{\circ} \mathrm{C}$ zwar die Geschwingigkeitskonstante zu, $k_{2}$ ist jedoch in Nitrobenzol nur $6.8 \mathrm{mal}$ grösser als im unpolaren Decan.

\section{Diskussion}

Das aufgefundene Geschwindigkeitsgesetz zweiter Ordnung lässt auf einen assoziativen Mechanismus schliessen. Damit stimmen auch die Aktivierungsparameter überein. Die jeweils ungewöhnlich niedrige Aktivierungsenthalpie und die stark negative Aktivierungsentropie deuten darauf hin, dass der Cycloadditionsschritt geschwindigkeitsbestimmend ist. Diese Folgerung wird durch die Ergebnisse der kinetischen Untersuchung der Reaktion von Selenoketon-Komplexen mit Dienen bestätigt [8]. Die Reaktionsprodukte sind in diesem Fall metallkoordinierte Selenacyclen (Gl. 3). Die Aktivierungsenthalpie $\Delta H^{*}$ für z.B. die Reaktion von Ib mit Cyclopentadien in Toluol beträgt $46(2) \mathrm{kJ} / \mathrm{mol}$, die Aktivierungsentropie $\Delta S^{\neq}$ $-147(5) \mathrm{J} /(\mathrm{mol} \mathrm{K})$.

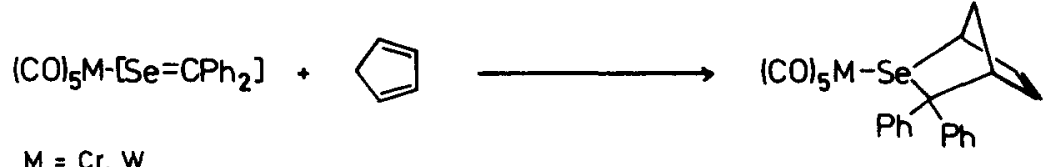


Die Werte für die Reaktion von Ib mit II in Toluol (siehe oben) liegen in der gleichen Grössenordnung. Der geschwindigkeitsbestimmende Schritt sollte somit bei beiden Reaktionen ähnlich sein. Dies bedeutet, dass die elektrocyclische Ringöffnung $(\mathbf{A} \rightarrow$ III, Gl. 2) im Vergleich zum geschwindigkeitslimitierenden Cycloadditionsschritt rasch erfolgen muss. In Übereinstimmung damit konnten bisher auch keine spektroskopischen Hinweise für ein intermediäres Auftreten von A bei der Reaktion von I mit II erhalten werden.

Mit zunehmender Fähigkeit der para-ständigen Substituenten $\mathbf{R}$ in $\mathrm{Ib}-\mathrm{e}$, die Elektronendichte im Ring und damit auch in der $\mathrm{Se}=\mathrm{C}$-Bindung zu erhöhen, nimmt die Geschwindigkeit der Cycloaddition deutlich ab. Die Geschwindigkeitskonstanten $k_{2}$ korrelieren sehr gut mit den Hammett-Konstanten $\sigma_{\mathrm{p}}$ für R. Die Reaktionskonstante $\rho$ ist mit 2.50 nahezu gleich den $\rho$-Werten für die $[4+2]$-Cycloaddition von Cyclopentadien an Ib-e $(\rho=2.48)$ [8] bzw. die Diels-Alder-Reaktionen von substituierten Nitrosobenzolen mit 2,3-Dimethylbuta-1,3-dien $(\rho=+2.50)$ [9] oder Cyclohexa-1,3-dien ( $\rho=2.57$ ) [10]. Dies bestätigt erneut, dass bei der Reaktion von G1. 2 der Additionsschritt ausschliesslich geschwindigkeitsbristimmend ist. Gleichzeitig weist die Ähnlichkeit der $\rho$-Werte auf die enge Verwandtschaft zwischen den Reaktionen von I mit II und normalen, unter $\mathrm{HOMO}_{\text {Dien }}-\mathrm{LUMO}_{\text {Dienophil }}-$ Kontrolle ablaufenden Diels-Alder-Reaktionen hin (zu Diels-Alder-Reaktionen siehe [11]).

Vergleichsweise klein ist der Einfluss des Zentralmetalls, möglicherweise kompensieren sich jedoch in diesem Fall sterische und elektronische Effekte weitgehend.

Auch der Solvenseffekt ist nur gering ausgeprägt. Im stark polaren Nitrobenzol (Dielektrizitätskonstante: 34.8 [12]) ist die Geschwindigkeitskonstante nur $6.8 \mathrm{mal}$ grösser als im wenig polaren Decan (Dielektrizitätskonstante: 2.0 [12]). Im Gegensatz dazu beobachtet man bei [2+2]-Cycloadditionen, die über einen dipolaren Übergangszustand bzw. eine dipolare Zwischenstufe ablaufen, beim Wechsel zu polaren Solvenzien eine drastische Reaktionsbeschleunigung (mit einem $k$ (Nitromethan)/ $k$ (Alkan)-Verhältnis von bis zu $10^{5}$ ) (Vergl. hierzu [13]). Ein stark dipolarer Übergangszustand kann damit für die $[2+2]$-Cycloaddition von II an I ausgeschlossen werden. Bei [4+2]-Cycloadditionen ist der Lösungsmitteleinfluss auf die Geschwindigkeit ebenfalls nur gering [11].

Bezüglich des geschwindigkeitsbestimmenden Schritts lässt sich die Umsetzung von I mit II somit am besten als konzertierte biradikaloide Reaktion mit einem frühen Übergangszustand beschreiben (vgl. hierzu auch [14]).

Aufschlussreich ist auch ein Vergleich der Reaktionen von 1-Diethylamino-1-propin (II) mit verschiedenen polaren Doppelbindungssystemen. Das Alkin II reagiert nicht nur mit der Se=C-Doppelbindung von I sondern auch mit der $\mathbf{M}=\mathrm{C}$-Doppelbindung von Carben-Komplexen (2.B. Gl. 4) [15], der S=C-Bindung von Diphenylthioketon (Gl. 5) [16] und der $\mathrm{O}=\mathrm{C}$-Bindung von Diphenylketen (Gl. 6) [17] unter Bildung des prinzipiell gleichen Produkttyps $\mathrm{X}=\mathrm{C}\left(\mathrm{NEt}_{2}\right) \mathrm{C}(\mathrm{Me})=\mathrm{CR}_{2}$.

Bezüglich der einzelnen Einflussgrössen (Abhängigkeit der Reaktionsgeschwindigkeit von den Ringsubstituenten, dem Zentralmetall und dem Solvens) sind die Reaktionen von II mit I (Gl. 2) bzw. Arylcarben-Komplexen (Gl. 4) [18] eng verwandt: die Reaktionsgeschwindigkeit nimmt in beiden Fällen mit zunehmender Donorfähigkeit der Ringsubstituenten deutlich ab ( $\rho$-Wert des Hammett-Plots: +2.50 (Gl. 2) bzw. +2.0 (Gl. 4)), wird hingegen von einem Wechsel des Zentralmetalls nur wenig beeinflusst $\left(k(\mathrm{~W}) / k(\mathrm{Cr})=3.48(\mathrm{Gl} .2), 3.38\left(\mathrm{Gl} .4\right.\right.$, bei $20^{\circ} \mathrm{C}$ in 


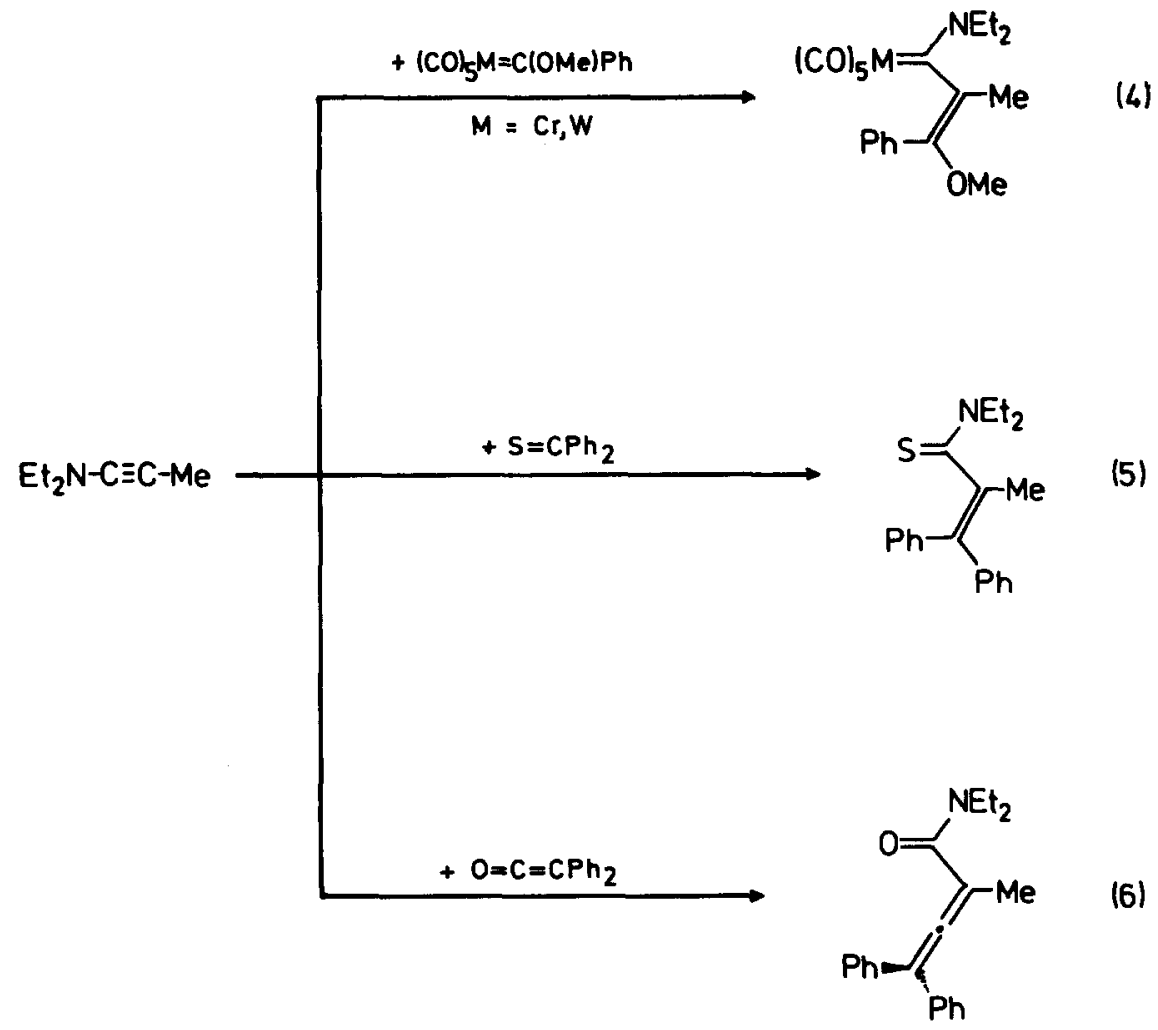

Octan)). Auch die Aktivierungsparameter sind für beide Umsetzungen gut miteinander vergleichbar: $\Delta H^{*}=32.7(1.7) \mathrm{kJ} / \mathrm{mol} ; \Delta S^{*}=-134(6) \mathrm{J} /(\mathrm{mol} \mathrm{K})$ (für $\mathrm{M}=\mathrm{W}$ und $\mathrm{R}=\mathrm{H}, \mathrm{Gl}$. 4) [18]. Die Umsetzung von II mit (CO) ${ }_{5} \mathrm{~W}=\mathrm{C}(\mathrm{OMe}) \mathrm{Ph}$ verläuft bei $20.6^{\circ}$ in Alkanen nur etwa halb so schnell.

Beträchtlich langsamer ist hingegen die Reaktion von II mit Diphenylthioketon (Gl. 5), für die eine zwitterionische Zwischenstufe vorgeschlagen wurde, kinetische Untersuchungen liegen jedoch nicht vor. Entsprechendes gilt für die Umsetzung von I mit Diphenylketen (G1. 6).

\section{Experimentelles}

Alle Arbeiten wurden unter $\mathrm{N}_{2}$-Schutz durchgeführt. Die Lösungsmittel waren sorgfältig getrocknet $\left(\mathrm{Na}\right.$, Molekularsieb $4 \AA$ ) und $\mathrm{N}_{2}$-gesättigt. Die Ausgangskomplexe I [5] und das verwendete 1-Diethylamino-1-propin [19] wurden nach Literaturangaben hergestellt.

\section{Kinetische Untersuchungen}

Die kinetischen Untersuchungen wurden auf VIS-spektroskopischem Weg (Cary 17D) durchgeführt. $2.5 \mathrm{ml}$ einer in der Küvette thermostatisierten Lösung von II im jeweiligen Solvens wurden rasch mit $0.5 \mathrm{ml}$ einer thermostatisierten Lösung der Komplexe I im gleichen Solvens versetzt. Die Küvette wurde verschlossen und geschüttelt, um eine gute Durchmischung der beiden Lösungen zu erreichen. Anschliessend wurde die Konzentration des Ausgangskomplexes über die Extink- 
tion der Reaktionslösung bei $630 \mathrm{~nm}$ (Ia) bzw. $586 \mathrm{~nm}$ (Ib-e) in Abhängigkeit von der Zeit kontinuierlich ermittelt. Die Temperatur wurde nach Ende der Umsetzung mit vorher geeichten Thermistoren betimmt (Genauigkeit: $\pm 0.1^{\circ} \mathrm{C}$ ). Die $\mathrm{Re}$ aktionen wurden jeweils über 8-10 Halbwertszeiten verfolgt. Die beim Auftragen von $\log \left(E_{t}-E_{\infty}\right)\left(E_{t}=\right.$ Extinktion zum Zeitpunkt $t, E_{\infty}=$ Extinktion nach Ende der Umsetzungen) gegen die Zeit $t$ resultierenden Kurven waren im Bereich von mindestens drei, im allgemeinen jedoch von vier bis fünf Halbwertszeiten linear (Korrelationskoeffizient besser als 0.9990). Die Geschwindigkeitskonstanten wurden unter Berücksichtigung der ersten drei Halbwertszeiten berechnet (jeweils ca. 150 $E_{t} / t$-Messwertepaare). Die in den Tabellen enthaltenen Werte sind Mittelwerte von mindestens zwei Messungen unter gleichen Bedingungen. Die Abweichung der Einzelmesswerte vom Mittelwert betrug maximal 3.0\% (Ausnahme: Abweichungen bis zu 5\% bei den Bestimmungen in 1,1,2-Trichlorethan). Der Korrelationskoeffizient für die Ermittlung der Aktivierungsparameter (Auftragung von $\ln \left(k_{2} / T\right.$ ) gegen $1 / T$ ) betrug -0.995 (Ia) bzw. -0.999 (Ib).

\section{Dank}

Wir danken der Deutschen Forschungsgemeinschaft und dem Fonds der Chemischen Industrie für die Förderung dieser Untersuchungen.

\section{Literatur}

1 H. Fischer und S. Zeuner, J. Organomet. Chem., 327 (1987) 63.

2 Vergleiche hierzu: (a) K.A. Jensen und A. Kjaer in S. Patai und Z. Rappoport (Hrsg.), The chemistry of organic selenium and tellurium compounds, Wiley, Chichester, 1986, S. 1; (b) P.D. Magnus in D.N. Jones (Hrsg.), Comprehensive Organic Chemistry Vol. 3, Pergamon Press, Oxford, 1979, S. 489; (c) H. Bock, A. Aygen, P. Rosmus, B. Solouki und E. Weissflog, Chem. Ber., 117 (1984) 187.

3 (a) G.A. Krafft und P.T. Meinke, J. Am. Chem. Soc., 108 (1986) 1314; (b) G.W. Kirby und A.N. Tretheway, J. Chem. Soc., Chem. Commun., (1986) 1152.

4 (a) C.E.L. Headford und W.R. Roper, J. Organomet. Chem., 244 (1983) C53; (b) L. Hofmann und H. Werner, J. Organomet. Chem., 255 (1983) C41; (c) H. Werner, L. Hofmann, J. Wolf und G. Müller, J. Organomet. Chem., 280 (1985) C55.

5 H. Fischer, S. Zeuner und J. Riede, Angew. Chem., 96 (1984) 707; Angew. Chem. Int. Ed. Engl., 23 (1984) 726.

6 H. Fischer, U. Gerbing, J. Riede und R. Benn, Angew. Chem., 98 (1986) 80; Angew. Chem. Int. Ed. Engl., 25 (1986) 78.

7 H. Fischer, A. Tiriliomis, U. Gerbing, B. Huber und G. Müller, J. Chem. Soc., Chem. Commun., im Druck.

8 H. Fischer, unveröffentlichte Untersuchungen.

9 M. Ahmad und J. Hamer, J. Org. Chem., 31 (1966) 2829.

10 G. Kresze, J. Firl, H. Zimmer und U. Wollnik, Tetrahedron, 20 (1964) 1605.

11 J. Sauer und R. Sustmann, Angew. Chem., 92 (1980) 773; Angew. Chem. Int. Ed. Engl., 19 (1980) 779.

12 R.C. Weast (Hrsg.), Handbook of Chemistry and Physics, CRC Press, Cleveland, 55th Edition, 1974, E57.

13 R. Huisgen, Acc. Chem. Res., 10 (1977) 117.

14 M.J.S. Dewar, S. Olivella und J.J.P. Stewart, J. Am. Chem. Soc., 108 (1986) 5771 und dort zitierte Literatur.

15 (a) K.H. Dötz und C.G. Kreiter, J. Organomet. Chem., 99 (1975) 309; (b) K.H. Dötz, Chem. Ber., 110 (1977) 78; (c) K.H. Dötz und I. Pruskil, Chem. Ber., 111 (1978) 2059; (d) K.H. Dötz, B. Fügen-Köster und D. Neugebauer, J. Organomet. Chem., 182 (1979) 489.

16 A.C. Brouwer und H.J.T. Bos, Tetrahedron Lett., (1976) 209.

17 M. Delaunois und L. Ghosez, Angew. Chem., 81 (1969) 33; Angew. Chem. Int. Ed. Engl., 8 (1969) 72.

18 H. Fischer und K.H. Dötz, Chem. Ber., 113 (1980) 193.

19 Houben-Weyl, Methoden der organischen Chemie, Band V/2a, Thieme, Stuttgart, 1977, S. 306. 